\title{
Cryptogenic Stroke Caused by a Carotid Web with a Superimposed Thrombosis: Serial Neurosonologic Findings
}

\author{
Jung-A Kwon (D), Dong Seok Gwak ${ }^{*}$ (D), Dong-Hyun Shim ${ }^{*}$ (D), Yong-Won Kim ${ }^{*} \dagger$ (D), Yang-Ha Hwang ${ }^{*} \dagger$ (D) \\ Department of Neurology, Kyungpook National University Hospital ${ }^{\star}$, Daegu; Department of Neurology, School of Medicine, Kyungpook \\ National University ${ }^{\dagger}$, Daegu, Korea
}

Recently, carotid web has been considered as an infrequent cause of cryptogenic stroke in young patients with no other identifiable stroke etiologies. It could lead to thrombus formation in the rostral aspects of the web, which subsequently provokes artery-to-artery embolism to the anterior circulation. A 48-year-old man who underwent endovascular mechanical thrombectomy for acute right middle cerebral artery occlusion was found to have a carotid web as the etiology of the index stroke. Owing to the presence of parenchymal hematoma after the thrombectomy, the administration of antithrombotics was withheld. Serial monitoring with carotid ultrasonography (CUS) revealed a reemergence of the superimposed thrombosis along the web, which necessitated urgent anticoagulation to prevent recurrent brain embolism. Therefore, serial monitoring with CUS could reveal valuable information regarding any presence of superimposed thrombosis on the web, which may revamp the preventive strategies in patients with acute stroke and carotid web.

\section{J Neurosonol Neuroimag 2019;11(2):15\$-161}

Key Words: Cerebral infarction; Carotid artery thrombosis; Ultrasonography; Fibromuscular dysplasia
Received: August 4, 2019

Revised: August 26, 2019

Accepted: August 30, 2019

Address for correspondence: Yang-Ha Hwang

Department of Neurology, Kyungpook National University Hospital, 130 Dongdeok-ro, Jung-gu, Daegu 41944, Korea Tel: $+82-53-420-5758$

Fax: +82-53-422-4265 E-mail: yangha.hwang@gmail. com
Carotid web is pathologically defined as an intimal variant of fibromuscular dysplasia, which appears radiographically as a shelf-like filling defect on the posterior aspect at the origin of the internal carotid artery (ICA) ${ }^{1-3}$ Available literature indicates that carotid web leads to ischemic stroke in young patients (aged $<60$ years) without any other cause of stroke. ${ }^{1,3}$ Hemodynamic changes such as blood flow stasis and turbulence in the rostral aspect of the carotid web result in thrombus formation and dislodgement, which can provoke artery-to-artery embolism to the anterior circulation. ${ }^{2}$ In the subanalysis of the multicenter randomized trial of endovascular treatment for acute ischemic stroke in the Netherlands (MR CLEAN) trial, the incidence of carotid web was $2.5 \%$ ipsilateral to index stroke. ${ }^{4}$ However, the serial imaging depiction of the carotid web was not well reported because of its rarity in clinical practice and the limited availability of repeated imaging acquisition. Herein, we report serial imaging findings of carotid web, using ultrasonography, which is the simplest and easily accessible imaging modality, to depict the natural course of the superimposed thrombosis in the carotid web with anticoagulant therapy.

\section{CASE REPORT}

A 48-year-old man was referred to Emergency Center of Kyungpook National University Hospital for the management of acute right middle cerebral artery (MCA) occlusion 240 minutes after stroke onset. At presentation, his National Institutes of Health Stroke Scale score was 17, which suggested clinical right 
MCA syndrome. Computed tomographic angiography of the supra-aortic vessels showed an occlusion of the proximal Mi segment of the right MCA (Fig. 1A) and a shelf-like filling defect arising from the posterior wall of the right proximal ICA, consistent with a carotid web (Fig. 1B). Diffusion-weighted imaging revealed increased signal intensities on the right basal ganglia and right frontotemporal areas, consistent with acute right MCA territorial infarcts (Fig. 1C). Considering his clinical and neuroimaging status, endovascular mechanical thrombectomy using the contact aspiration technique was performed, and complete reperfusion of the target MCA was achieved after 310 minutes from symptom onset without any procedure-related complications (Fig. 1D, E). ${ }^{5}$ Oneday follow-up brain computed tomography revealed hyperdensities with surrounding rim hypodensities on the right basal ganglia, which suggested parenchymal hematoma after reperfusion therapy (Fig. 1F). Owing to increased concern for hematoma expansion, no antithrombotics were administered until resolution of the hemorrhage.

To investigate the etiology of brain embolism, blood tests and analysis for hypercoagulable states and vasculitis, electrocardiography (ECG), continuous ECG monitoring, and transthoracic echocardiography were performed, but the results were unremarkable except for elevated D-dimer level (1.19 $\mu \mathrm{g} / \mathrm{mL})$. In addition, his medical history was unremarkable, but he currently smoked (20 pack-years). With the assumption that right carotid web could be the sole etiology of current stroke, carotid ultrasonography (CUS) was per-
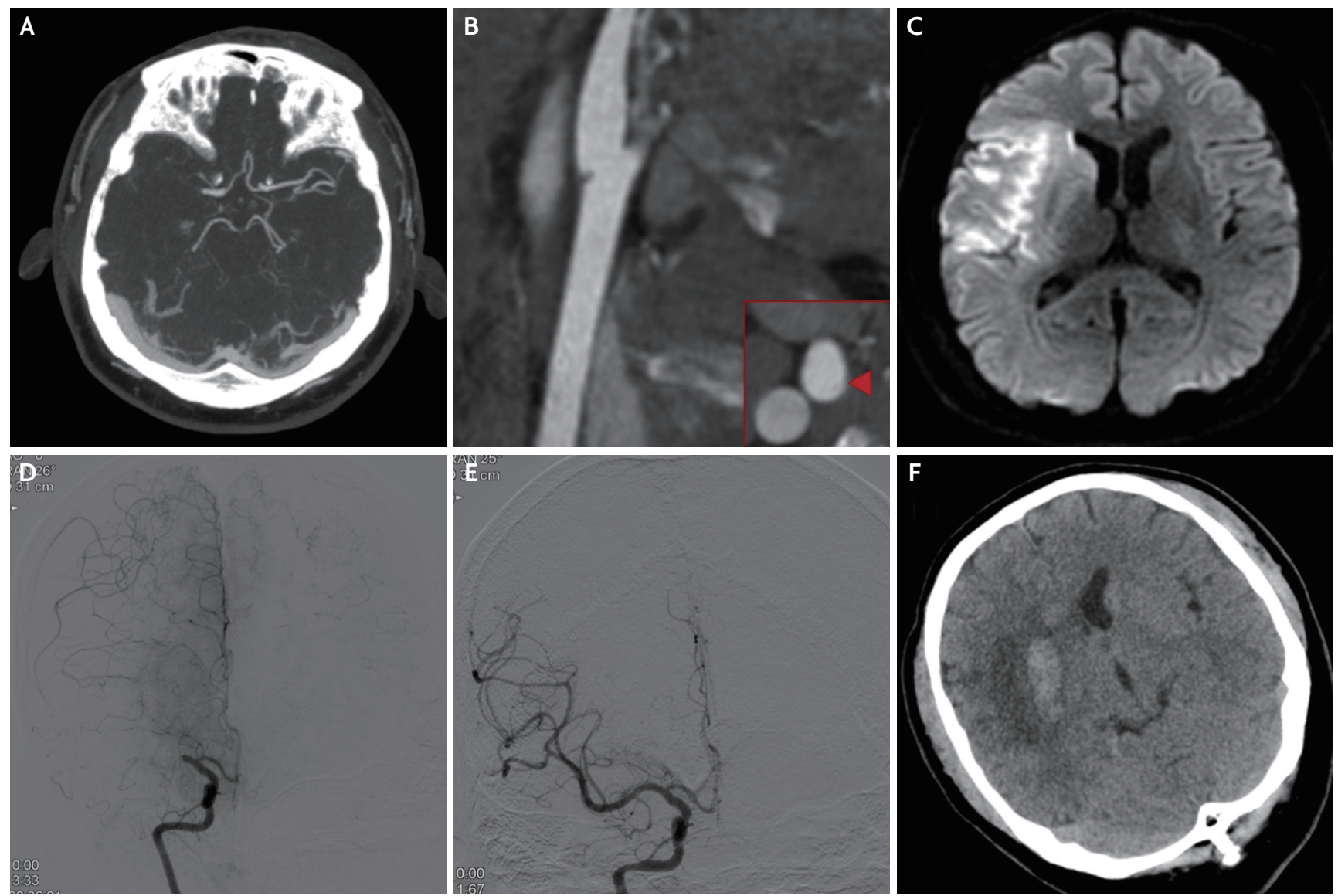

FIG. 1. Serial neurovascular imaging findings. At presentation, the initial computed tomographic angiogram of the supra-aortic vessels showed (A) acute occlusion of the right middle cerebral artery (MCA) and (B) a carotid web (shelf-like filling defect) arising from the posteromedial wall (arrowhead) of the right proximal internal carotid artery. (C) The diffusion-weighted image shows increased signal intensities on the right basal ganglia and right frontotemporal areas. The transfemoral cerebral angiogram confirms (D) the right MCA occlusion and shows (E) the subsequent complete reperfusion achieved using the contact aspiration technique. The 1-day follow-up brain computed tomography image shows (F) hyperdensities with surrounding rim hypodensities on the right basal ganglia. 
formed to monitor any presence of thrombosis around the web on day 4 after the index stroke. However, it revealed a hyperechoic thin web-like projection into the lumen without any evidence of thrombosis (Fig. 2A). After 8 days of admission, follow-up CUS was performed, which revealed a newly formed hypoechoic thrombus around the web (Fig. 2B, C, Supplementary Video 1). With thoughtful consideration of the risk of hematoma expansion and recurrent embolism, anticoagulation with low-molecular-weight heparin (fraxiparin 2,850 IU/0.3 mg twice daily) bridged with warfarin (target international normalized ratio, 2.0-3.0) was started on day 8. After 15 days of admission, a carotid web without any evidence of residual thrombosis was observed during the carotid ultrasonography (Fig. 2D). Thereafter, a carotid web without any thrombosis still remained on the 1- and 3-month follow-up CUS (Fig. 2E, F). At 3-month follow-up, his neurological status was much improved, enabling him to walk without any assistance.

\section{DISCUSSION}

Herein, we describe a case of acute ischemic stroke due to right MCA occlusion with an ipsilateral carotid web. From a clinical perspective, the following important points in our case deserve discussion: 1) a carotid web without any evidence of cardioembolism or large artery atherosclerosis could be the sole source of brain embolism, which necessitates endovascular mechanical thrombectomy; 4 2) a carotid web after acute ischemic stroke could be a source of recurrent embolism with reemergence of newly formed thrombus around the web, especially in cases not treated with antithrombotics during the acute phase of stroke because of the risk of hemorrhage; and 3) serial follow-up neurosonologic examinations, which are simple and readily accessible, could be an important diagnostic tool for decision making on the timing of antithrombotic use because a carotid web has an early dynamic course from innocent web to superimposed thrombosis.

Serial neurosonologic examinations can reveal the dynamic imaging features of carotid web and provide more-precise information on the morphological change of the superimposed thrombosis after anticoagulant therapy. However, only few descriptions of a carotid web observed using CUS have been reported in the literature, ${ }^{6}$ and the diagnostic sensitivity of CUS is
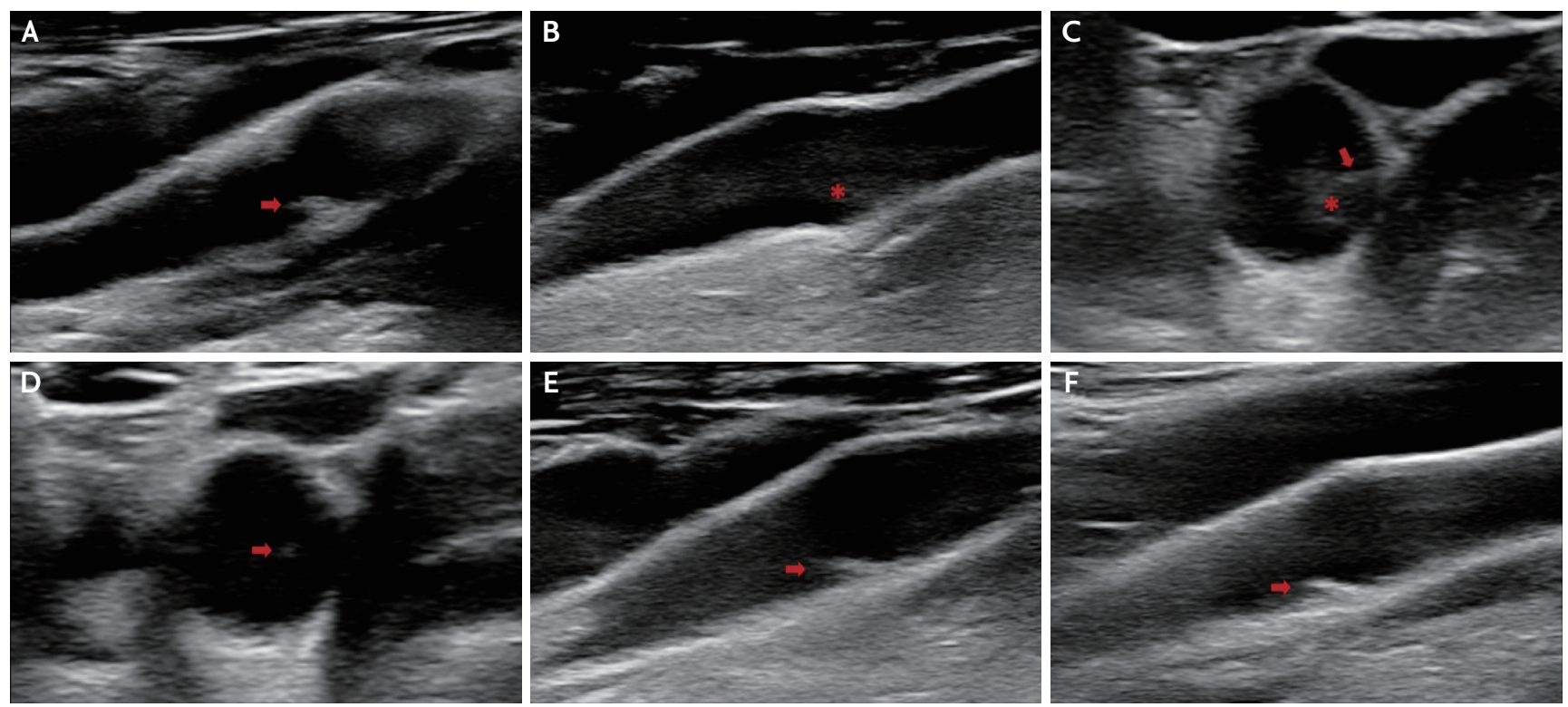

FIG. 2. Serial neurosonologic findings. Four days after the index stroke, a B-mode ultrasonography image showed (A) a hyperechoic web-like material projecting into the lumen (arrow) without any visible thrombosis. Eight days after the index stroke, a B-mode ultrasonography image showed $(B, C)$ hypoechoic components (asterisk) around the web (arrow), which suggested newly formed thrombosis in the longitudinal and transverse views. Fifteen days after the index stroke, a B-mode ultrasonography image showed (D) a carotid web (arrow) without any thrombosis in the transverse view. At 1 and 3 months after the index stroke, a B-mode ultrasonography image showed (E, F) a carotid web (arrow) without any thrombosis with concurrent anticoagulant therapy. 
limited by the small size and poor awareness of carotid web. The relatively common finding of carotid web was mild bulbar outgrowth. ${ }^{7}$ Reported ultrasonography findings of carotid web include a hypoechoic mass with a linear band extending into the lumen, ${ }^{8}$ a focally arising artifact and elevated peak systolic velocity, ${ }^{6}$ and irregular echogenic plaque resulting in stenosis. ${ }^{9}$ In our case, we detected a hyperechoic thin web-like projection into the lumen. In addition, we observed a subsequent appearance of hypoechoic thrombi around the web. These findings corroborate the proposed mechanism of carotid web causing hemodynamic change and subsequent thrombosis. In summary, on CUS imaging, a carotid web may appear as a characteristic thin strand protruding into the lumen, which can cause hemodynamic instability, with or without a superimposed thrombosis.

The recommended treatment strategies for patients with carotid web remain mostly unknown owing to its rarity and lack of natural history data. ${ }^{3}$ General medical management strategies, including antithrombic administration and optimal control of vascular risk factors, are considered traditional. However, considering the imaging findings of blood stasis or turbulence around the web, and the high incidence rate of superimposed thrombosis, which has been reported to range from $12 \%$ to $29 \%$ in patients with stroke attributable to web, ${ }^{1}$ anticoagulant therapy could be a reasonable alternative for secondary stroke prevention. Moreover, long-term therapy with anticoagulants could be needed in cases with fibrotic tissue carotid web without regression. In patients with failure of medical management, revascularization with either endovascular stenting or carotid endarterectomy may be considered. Recent case reports showed favorable results from stenting. ${ }^{10}$

In cases with acute ischemic stroke and carotid web without any other determined etiology of brain embolism, carotid web could serve as a nidus for thrombus formation and possibly a source of brain embolism. This condition can be serially monitored using CUS. Furthermore, reemergence of a newly formed thrombus along the carotid web could change the management strategies to prevent recurrent brain embolism. Future studies with large numbers of cases with carotid web could elucidate the natural course and identify more-definitive treatment strategies in these cases.

\section{SUPPLEMENTARY MATERIALS}

Supplementary materials related to this article can be found online at https://doi.org/10.31728/jnn.2019.00057.

\section{Conflicts of Interest}

No potential conflicts of interest relevant to this article was reported.

\section{REFERENCES}

1. Kim SJ, Nogueira RG, Haussen DC. Current understanding and gaps in research of carotid webs in ischemic strokes: a review. JAMA Neurol. 2019;76:355-361.

2. Choi PM, Singh D, Trivedi A, Qazi E, George D, Wong J, et al. Carotid webs and recurrent ischemic strokes in the era of CT angiography. AJNR Am J Neuroradiol. 2015;36:2134-2139.

3. Park HK, Hong KS. Carotid web: under-recognized etiology for ischemic stroke. J Neurosonol Neuroimag. 2018;10:100-105.

4. Compagne KCJ, van ES ACGM, Berkhemer OA, Borst J, Roos YBWEM, van Oostenbrugge RJ, et al. Prevalence of carotid web in patients with acute intracranial stroke due to intracranial large vessel occlusion. Radiology. 2018;286:10001007.

5. Kang DH, Hwang YH. Frontline contact aspiration treatment for emergent large vessel occlusion: a review focused on practical techniques. J Stroke. 2019;21:10-22.

6. Fu W, Crockett A, Low G, Patel V. Internal carotid artery web: Doppler ultrasound with CT angiography correlation. J Radiol Case Rep. 2015;9:1-6.

7. Joux J, Boulanger M, Jeannin S, Chausson N, Hennequin JL, Molinié V, et al. Association between carotid bulb diaphragm and ischemic stroke in young Afro-Caribbean patients: a population-based case-control study. Stroke. 2016;47:2641-2644.

8. Kliewer MA, Carroll BA. Ultrasound case of the day. Internal carotid artery web (atypical fibromuscular dysplasia). Radiographics. 1991;11:504-505.

9. Smyth H, Byrne D, Hayden D, Eoin K, Murphy S. A cause of recurrent strokes: carotid webs detected by CT angiogram. BJR Case Rep. 2017;4:20170066.

10. Haussen DC, Grossberg JA, Bouslama M, Pradilla G, Belagaje S, Bianchi N, et al. Carotid web (intimal fibromuscular dysplasia) has high stroke recurrence risk and is amenable to stenting. Stroke. 2017;48:3134-3137. 